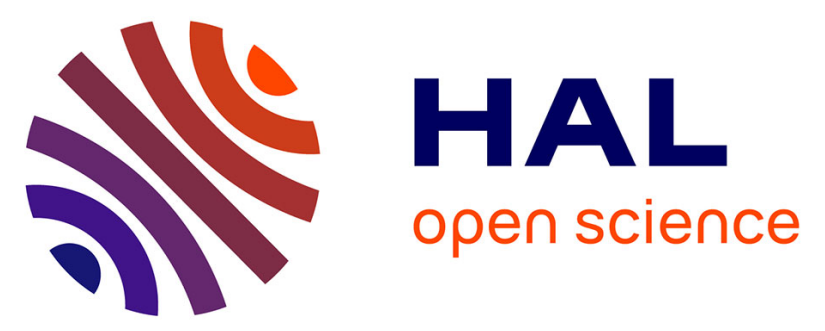

\title{
COMPETING MECHANISMS OF MOLECULAR HYDROGEN FORMATION IN CONDITIONS RELEVANT TO THE INTERSTELLAR MEDIUM
}

Jean-Louis Lemaire, G. Vidali, S. Baouche, M. Chehrouri, H. Chaabouni, H. Mokrane

\section{To cite this version:}

Jean-Louis Lemaire, G. Vidali, S. Baouche, M. Chehrouri, H. Chaabouni, et al.. COMPETING MECHANISMS OF MOLECULAR HYDROGEN FORMATION IN CONDITIONS RELEVANT TO THE INTERSTELLAR MEDIUM. The Astrophysical journal letters, 2010, 725, pp.L156-L160. hal00575649

\section{HAL Id: hal-00575649 \\ https://hal.science/hal-00575649}

Submitted on 10 Mar 2011

HAL is a multi-disciplinary open access archive for the deposit and dissemination of scientific research documents, whether they are published or not. The documents may come from teaching and research institutions in France or abroad, or from public or private research centers.
L'archive ouverte pluridisciplinaire HAL, est destinée au dépôt et à la diffusion de documents scientifiques de niveau recherche, publiés ou non, émanant des établissements d'enseignement et de recherche français ou étrangers, des laboratoires publics ou privés. 


\title{
COMPETING MECHANISMS OF MOLECULAR HYDROGEN FORMATION IN CONDITIONS RELEVANT TO THE INTERSTELLAR MEDIUM
}

\author{
J. L. Lemaire ${ }^{1}$, G. Vidali $^{1,3}$, S. Baouche ${ }^{1}$, M. Chehrouri ${ }^{1,2}$, H. ChaAbouni $^{1}$, ANd H. Mokrane ${ }^{1}$ \\ ${ }^{1}$ LAMAp/LERMA, UMR8112 du CNRS, de l'Observatoire de Paris et de l'Université de Cergy Pontoise, 5 mail Gay-Lussac, 95000 Cergy Pontoise Cedex, France; \\ jean-louis.lemaire@obspm.fr \\ ${ }^{2}$ LEPC Université de Saida, BP138, ENSAR, 20002 Saida, Algerie \\ Received 2010 September 25; accepted 2010 November 8; published 2010 November 29
}

\begin{abstract}
The most efficient mechanism of the formation of molecular hydrogen in the current universe is by association of hydrogen atoms on the surface of interstellar dust grains. The details of the processes of its formation and release from the grain are of great importance in the physical and chemical evolution of the space environments where it takes place. The main puzzle is still the fate of the $4.5 \mathrm{eV}$ released in $\mathrm{H}_{2}$ formation and whether it goes into internal energy (rovibrational excitation), translational kinetic energy, or heating of the grain. The modality of the release of this energy affects the dynamics of the ISM and its evolution toward star formation. We present results of the detection of the rovibrational states of the just-formed $\mathrm{H}_{2}$ as it leaves the surface of a silicate. We find that rovibrationally excited molecules are ejected into the gas phase immediately after formation over a much wider range of grain temperatures than anticipated. Our results can be explained by the presence of two mechanisms of molecule formation that operate in partially overlapping ranges of grain temperature. A preliminary analysis of the relative importance of these two mechanisms is given. These unexpected findings, which will be complemented with experiments on the influence of factors such as silicate morphology, should be of great interest to the astrophysics and astrochemistry communities.
\end{abstract}

Key words: astrochemistry - ISM: atoms - ISM: molecules - methods: laboratory - molecular processes

\section{INTRODUCTION}

As the most abundant molecule in the universe, hydrogen influences both the way stars are formed and the chemical evolution of interstellar clouds. Although it was recognized long ago that the formation of molecular hydrogen most likely occurs on dust grains (Hollenbach \& Salpeter 1971), as the radiative recombination involves spin-forbidden transition from the protomolecule to the ground state, it is only in the last decade that experiments were done aimed at understanding the mechanism and measuring the rates of $\mathrm{H}_{2}$ formation on dust grain analogs in simulated space environments (Pirronello et al. 1997).

The energy that the nascent molecule brings into the interstellar medium (ISM) is of interest in the overall evolution of space environments and in programs of detection of excited $\mathrm{H}_{2}$ in space. Measurements of the translational energy of HD from amorphous water-ice showed that the just-formed HD can carry energy into the ISM and could be detected (Manicò et al. 2001). The observed abundance of warm $\mathrm{H}_{2}$ gas in outer layers of photodissociation regions (PDRs) is hard to explain with current models, and contributions of excited $\mathrm{H}_{2}$ from grains have been invoked (Habart et al. 2004). Searches of rovibrational excitation of $\mathrm{H}_{2}$ that could be ascribed to "UV formation pumping" in PDR and dense clouds yielded no positive detection (Tiné et al. 2003; Lemaire \& Field 2001; Thi et al. 2009).

Laboratory studies can help observational searches by assessing how the energy is distributed and by characterizing rovibrational excitations upon $\mathrm{H}_{2}$ formation so to distinguish it from other excitation mechanisms, such as UV excitation, collisions, and shocks.

Results of experiments of $\mathrm{H}_{2}$ formation on surfaces of polycrystalline and amorphous silicates (Pirronello et al. 1997;

\footnotetext{
3 Current address: Physics Department, Syracuse University, Syracuse, NY 13244, USA
}

Vidali et al. 2007, 2009), amorphous water-ice (Roser et al. 2003; Hornekær et al. 2003; Amiaud et al. 2007), highly oriented pyrolitic graphite (HOPG; Islam et al. 2007; Latimer et al. 2008), and amorphous carbon (Katz et al. 1999) are consistent with $\mathrm{H}_{2}$ formation via the Langmuir-Hinshelwood (L-H) mechanism or possibly the Harris-Kasemo (H-K) "hot atom" mechanism (Harris \& Kasemo 1981), at least when the number of atoms on the surface is small. While the L-H mechanism is known to occur in the vast majority of chemical reactions at surfaces, the other involves the superthermal motion of atoms as they land on the surface. In this case, atoms are not accommodated by the surface, and the new molecule can carry substantial energy in translational and rovibrational excitations. Another mechanism, the Eley-Rideal reaction, consists in the direct reaction, without prior energy accommodation, of an atom from the gas phase with one on the surface. This mechanism is operative in $\mathrm{H}_{2}$ / $\mathrm{HD}$ formation in H-loaded amorphous carbon (Mennella 2008). Photodissociation of amorphous solid water also involves other mechanisms to yield both cold and hot $\mathrm{H}_{2}$ products (Yabushita et al. 2008).

Most of the prior work on $\mathrm{H}_{2}$ formation via the H-K mechanism was done on metals and semiconductors, where there is large condensation energy (Kammler et al. 2000). It has not been established whether or how the $\mathrm{H}-\mathrm{K}$ mechanism operates in $\mathrm{H}_{2}$ formation on dust grain analogs at low temperature $(<20 \mathrm{~K})$. Experiments showed that rovibrationally hot molecules formed on ice leave the surface de-excited (Congiu et al. 2009). On HOPG (Islam et al. 2007; Latimer et al. 2008; Zecho et al. 2002), a large fraction of molecules desorb in the $v=4$ state, but in this case the measurements were done at higher sample temperature ( $>15 \mathrm{~K}$ ) than in the other studies. This leads to two pressing questions: (1) whether and how silicates, which are a major constituent of interstellar dust and shown to catalyze $\mathrm{H}_{2}$ formation at low temperature via the L-H mechanism (Vidali et al. 2009), are also involved in the formation of highly excited $\mathrm{H}_{2}$; and (2) over which grain temperature range the formation 


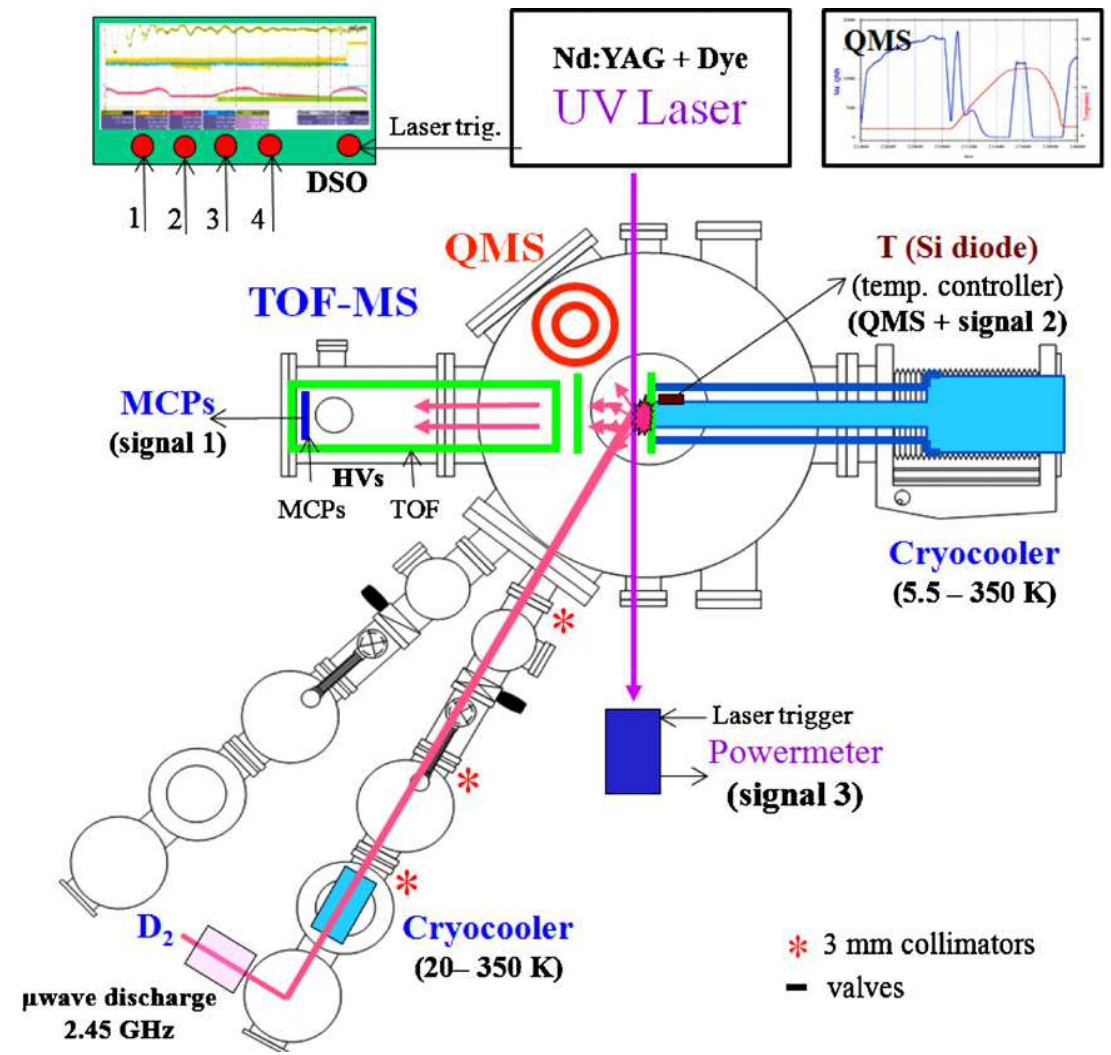

Figure 1. Schematic of the "FORMOLISM" (FORmation of MOLecules in the InterStellar Medium) apparatus used for these experiments.

of excited $\mathrm{H}_{2}$ occurs, since this is relevant to understanding $\mathrm{H}_{2}$ formation in ISM regions where the $\mathrm{L}-\mathrm{H}$ mechanism does not work because of the high temperature of grains (Habart et al. 2004; Cazaux \& Tielens 2004).

We report here the detection of rovibrationally hot $\mathrm{D}_{2}$ formed on the surface of a silicate (deuterium is used because hydrogen is the main background gas in a well-baked ultra-high vacuum apparatus); we show that it occurs in a surface temperature range that, while consistent with the weak interaction between D and the silicate, is much wider than thermal programmed desorption (TPD) experiments indicated (Perets et al. 2007). We propose that it must be ascribed to the $\mathrm{H}-\mathrm{K}$ mechanism competing with the $\mathrm{L}-\mathrm{H}$ mechanism in the formation of $\mathrm{D}_{2}$.

\section{EXPERIMENTAL METHODS AND RESULTS}

Experiments are performed using the setup of Figure 1. We summarize here the major features; details are given in Baouche (2004), Amiaud (2006), and Matar (2009).

The apparatus consists of an ultra-high vacuum stainless steel chamber $\left(<10^{-10}\right.$ mbar $)$. At its center, a sample holder is thermally connected to the cold finger extension of a closedcycle He cryostat. The temperature (in the 5.5-350 K range) is measured with two calibrated Si diodes, one clamped on the sample and another on the tip of the second stage of the cryostat where a resistive heater is located. In a TPD, the combination of the length and cooling power of the cold finger yields a delayed desorption of gases adsorbed on the sample supports. This allows us to separate the contribution of the sample of the one from other parts of the cold finger. An amorphous olivinetype silicate film was kindly provided by Dr. D'Hendecourt (IAS Orsay) by thermal evaporation of San Carlos olivine (Djouadi et al. 2005) onto a gold-coated substrate. Its properties have been checked by infrared spectroscopy on a $\mathrm{KBr}$ substrate coated by a silicate film at the same time as the copper sample (Davoisne et al. 2008).

The atomic jet is prepared in a microwave cavity in a triply differentially pumped line. The beam is bent in a teflon tube connected to a thick aluminum cone-shaped nozzle attached to a closed-cycle $\mathrm{He}$ cryostat. In this way, we ensure that no light or excited molecules created in the plasma reach the surface. We checked that no excited molecules are detected in the beam, either at room temperature or at $50 \mathrm{~K}$. The experimentally estimated $\mathrm{D}_{2}$ beam flux is $\sim 9 \times$ $10^{12}$ molecules $\mathrm{cm}^{-2} \mathrm{~s}^{-1}$. The $\mathrm{D}_{2}$ dissociation is checked regularly during the experiments (typically $80 \%$ ), as measured by a quadrupole mass spectrometer (QMS). During the irradiation phase, the sample is exposed to $\sim 1.35 \times 10^{13} \mathrm{D}$-atoms cm $\mathrm{cm}^{-2} \mathrm{~s}^{-1}$ (Amiaud et al. 2007; Accolla et al. 2010). The QMS is moved above the sample to avoid interferences with the time-of-flight (TOF) spectrometer and to record the background pressure during both deposition and TPD experiments. TPDs are performed using a $10 \mathrm{~K}_{\text {minute }}{ }^{-1}$ linear heating ramp, and the $\mathrm{D}_{2}, \mathrm{HD}$, and $\mathrm{H}_{2}$ signals are recorded.

To measure the rovibrational excitation of the molecules leaving the surface, we used resonantly enhanced multi-photon ionization (REMPI) coupled with TOF mass spectroscopy. A UV laser beam is tuned to ionize and detect selectively a given rovibrational state via 2-photon absorption through a virtual state followed by ionization: $(2+1)$ REMPI. In this way, we sample the population of $\mathrm{D}_{2}$ formed in the $v^{\prime \prime}=$ $4, J^{\prime \prime}=2$ rovibrationally excited state of the ground state. This transition has been chosen for its suitable Franck-Condon factor (Fantz \& Wunderlich 2004). A tunable dye laser pumped with the second harmonic of an ND:YAG laser $(2 \mathrm{~J} / 20 \mathrm{~Hz})$ followed by frequency mixing gives $\sim 222.6 \mathrm{~nm}$ photons with 


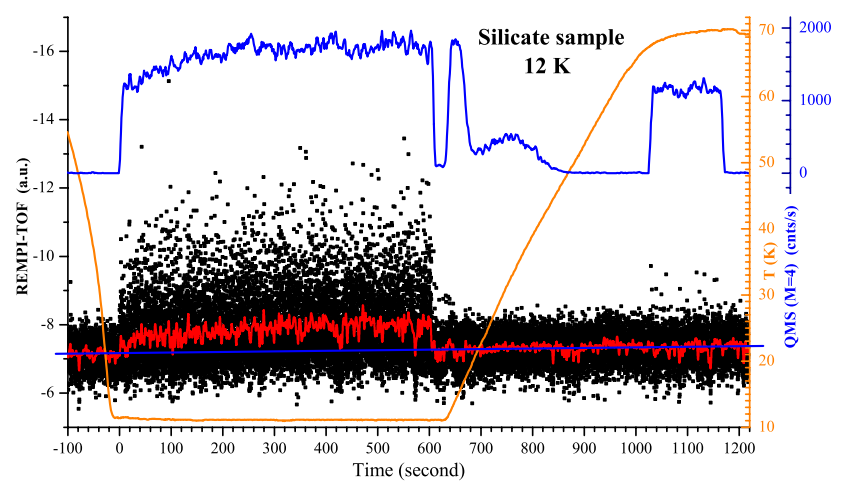

Figure 2. REMPI-TOF signal (in black; in red, 25-point FFT filtering) shows the formation of $\mathrm{D}_{2}$ in the $v^{\prime \prime}=4, J^{\prime \prime}=2$ excited state when irradiating the sample at $12 \mathrm{~K}$ with $\mathrm{D}$ atoms from time $t=0$ to $600 \mathrm{~s}$. Blue (scale offset): QMS $\mathrm{D}_{2}$ signal; its rise at $t=0$ is due to the increased gas load from the beam. Orange: sample temperature. During the temperature ramp of the TPD (beam valve closed), a sharp peak of $\mathrm{D}_{2}$ molecules desorbing from the surface is seen whereas no signal appears on the REMPI (the second broad and smaller peak during the TPD is an artifact due the cryostat, see the text). When the temperature reaches $70 \mathrm{~K}$, the irradiation resumes. The QMS signal goes to the same level as at $t=0$ (which is due to the extra $\mathrm{D}_{2}$ gas load from the beam) while the REMPI signal is almost negligible.

200-300 $\mu \mathrm{J}_{\text {pulse }}{ }^{-1}$. The laser beam is focused $\sim 4 \mathrm{~mm}$ away from the center of the surface. At the exit of the main chamber, the shot-by-shot laser pulse integrated power appears rather stable $(\sim 7 \%)$ over a few hours. The REMPI-TOF signal is recorded and integrated. Correcting the REMPI-TOF data using a power law to account for its nonlinear dependence with laser power made little difference, as the main source of noise is the random desorption of excited molecules.

An ancillary setup is composed of a hot molecular hydrogen lamp and a TOF spectrometer with characteristics very similar to the one in the main apparatus (Malmasson 1994). The source provides rovibrationally excited states (Schermann et al. 1994) by dissociating hydrogen on a $2900 \mathrm{~K}$ tungsten filament followed by recombination and thermalization on the cool walls. The laser light is diverted to this setup for wavelength adjustment and REMPI signal intensity calibration.

The experiments are conducted as follows: once the silicate has reached a given temperature, it is irradiated with a beam of $\mathrm{D}$ and some undissociated $\mathrm{D}_{2} . \mathrm{D}_{2}, \mathrm{HD}$, and $\mathrm{H}_{2}$ signals are detected by the QMS while the REMPI-TOF simultaneously measures the population in a given $\mathrm{D}_{2}$ state. After 10 minutes of irradiation ( 5 minutes for temperatures $>16 \mathrm{~K}$ ), the separation valve is closed and immediately after, a TPD is started using the same diagnostics. We investigated the formation and excitation of $\mathrm{D}_{2}$ molecules at 13 sample temperatures between 5.5 and $70 \mathrm{~K}$. The REMPI and QMS signals obtained when the sample is irradiated at $12 \mathrm{~K}$ and then brought to $70 \mathrm{~K}$ are shown in Figure 2. We observe the following main features: (1) rovibrationally excited molecules are formed as soon as the sample is irradiated and (2) desorption in the TPD experiment yields no REMPI signal in the probed state. This means that there are no highly excited molecules leaving the surface during the TPD. Figure 3 shows the REMPI and QMS signals during D irradiation with the sample at $36 \mathrm{~K}$. Surprisingly, we still detect the formation of $\mathrm{D}_{2}$ in an excited state at this temperature, while there is no QMS-TPD signal because the residence time of atoms is too short. This is in agreement with experiments of $\mathrm{HD}$ formation on amorphous silicates using $\mathrm{H}$ and $\mathrm{D}$ beams (Vidali et al. 2009), where there is little or no TPD signal of HD after irradiation at sample temperature $>28 \mathrm{~K}$. At $70 \mathrm{~K}$, there

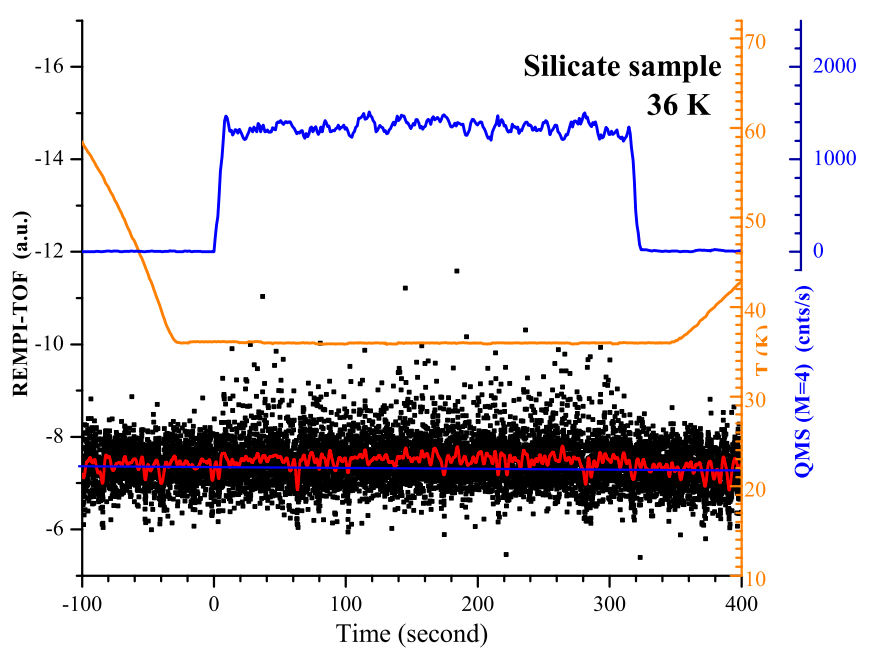

Figure 3. Same as Figure 2 with the sample at $36 \mathrm{~K}$ during irradiation. There is no TPD-QMS signal of $\mathrm{D}_{2}$ from the surface during the temperature ramp $(t>$ $360 \mathrm{~s}$ ) as no deuterium stick on the surface at such temperature.

is almost no detectable REMPI signal during irradiation (see Figure 2).

Figure 4 shows the integrated REMPI and TPD signals for $\mathrm{D}$ irradiation at different sample temperatures. The maximum formation efficiency in both cases is at $T \sim 10 \mathrm{~K}$. From this figure we find that (1) the rovibrationally excited molecules are detected over a range of sample temperature that is considerably wider than the one for the TPD signal. The decay of the REMPI signal versus sample temperature at irradiation is markedly different from the TPD signal, pointing to different mechanisms of $\mathrm{D}_{2}$ formation (see below). (2) For $T<10 \mathrm{~K}$, there is a diminishing REMPI signal while the QMS signal is slightly lower only at $5.5 \mathrm{~K}$. This corresponds to a more efficient accommodation of the incoming atoms and to a slower diffusion time on the surface. Both the QMS and REMPI signals increase over time since the beginning of the irradiation (see Figure 2). This is also observed at temperatures up to $18 \mathrm{~K}$ where the signal quickly reaches a constant value. This is due to the changing sticking coefficient of D atoms as the fraction of the surface covered by $\mathrm{D}$ and $\mathrm{D}_{2}$ increases. At high temperature (see Figure 3), the REMPI signal reaches steady state immediately. Experiments of $\mathrm{D}_{2}$ sticking on a single crystal forsterite show that the sticking probability has a sharp transition at the sample temperature of 15-16 K (P. Frank et al. 2010, private communication).

\section{ANALYSIS OF THE EXPERIMENTAL RESULTS}

At $P=4 \times 10^{-4}$ mbar in the test chamber, there are $\sim 1.1 \times$ $10^{13}$ molecules $\mathrm{cm}^{-3}$ in all vibrational and rotational states. Using $5.8 \times 10^{-3}$ as the ratio of the populations in $v^{\prime \prime}=4$, (all $J^{\prime \prime}$ ) and in all states (Schermann et al. 1994), we get $6.4 \times$ $10^{8}$ molecules $\mathrm{cm}^{-3}$ in $v^{\prime \prime}=4$. The ratio of TOF-REMPI $v^{\prime \prime}=4, J^{\prime \prime}=2$ signals in the ultra-high vacuum chamber (sample at $12 \mathrm{~K}$ ) and in the test chamber is $6.3 \times 10^{-3}$.

For the population of $\mathrm{D}_{2}$ desorbing from the silicate at $12 \mathrm{~K}$ in $v^{\prime \prime}=4$, the $J^{\prime \prime}$ excited state has $6.4 \times 10^{8} \times\left(6.3 \times 10^{-3}\right)=$ $4 \times 10^{6}$ molecules $\mathrm{cm}^{-3}$. Of these, $1.1 \times 10^{6}$ molecules $\mathrm{cm}^{-3}$ are in the $v^{\prime \prime}=4, J^{\prime \prime}=2$ state, where for lack of other data, we used the results for HD on graphite at $15 \mathrm{~K}$ (Islam et al. 2007): of the molecules in the $\left(v^{\prime \prime}=4\right.$, all $\left.J\right)$ states $27.5 \%$ are in $J^{\prime \prime}=2$. 


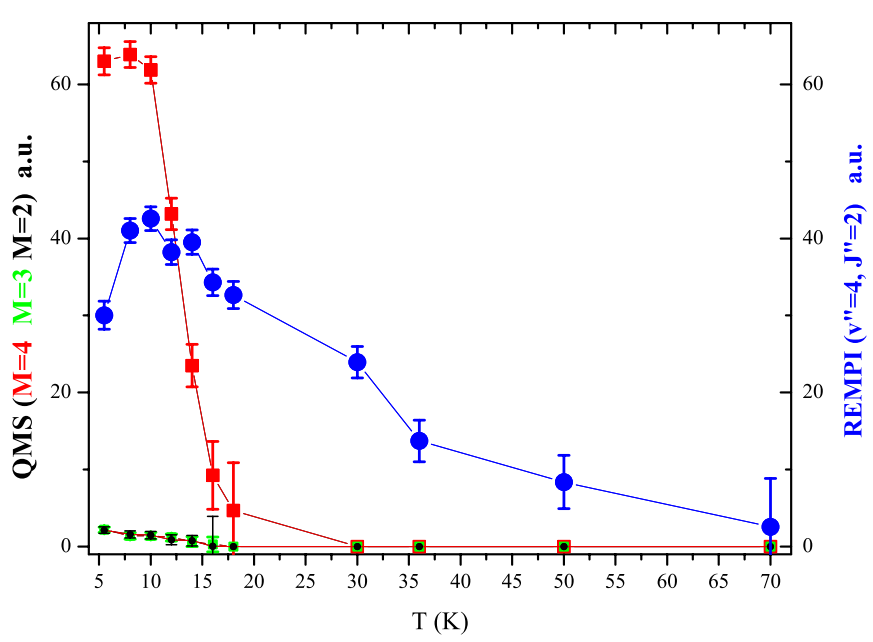

Figure 4. Integrated intensities of the REMPI $\left(\mathrm{D}_{2}\right)$ signal during irradiation and of the QMS $\left(\mathrm{D}_{2}\right)$ signal of the TPD after irradiation vs. the sample temperature during irradiation. The HD signal is due to exchange reactions on the sample and/or walls of the apparatus.

The density in all $(v, J)$ states in the incoming beam is given by flux/velocity $\left(v=5.6 \times 10^{4} \mathrm{~cm} \mathrm{~s}^{-1}\right.$ at $\left.50 \mathrm{~K}\right)$. The $\mathrm{D}_{2}$ flux reported above gives, at $80 \%$ dissociation, $7.2 \times 10^{12}$ molecules $\mathrm{cm}^{-2} \mathrm{~s}^{-1}$ and a density of $\sim 1.3 \times 10^{8}$ molecules $\mathrm{cm}^{-3}$, where we assumed that all atoms stick and recombine on the sample. This is the highest number of molecules that can be formed by whatever mechanism. In the case where they all formed by the $\mathrm{H}-\mathrm{K}$ mechanism and assuming that they leave the surface in any direction, the number density that could be detected depends on the size of the laser's active region for ionization. Assuming a Gaussian beam, the confocal parameter $b=7 \mathrm{~cm}$ and the beam waist $2 w_{0}=100 \mu \mathrm{m}$. From the beam waist the surface is seen under an angle of $\sim 41^{\circ}$, allowing us to detect $41 / 180$ of the molecules and a density of $\sim 2.9 \times 10^{7}$ molecules $\mathrm{cm}^{-3}$ in all states (including $v^{\prime \prime}=0$ ). Referring again to the results of Latimer et al. (2008) at $15 \mathrm{~K}$, assuming the same ratio of $46 \%$ of $v^{\prime \prime}=4$ versus all $v^{\prime \prime}=1-7$ for silicates, we get $\sim 8.7 \times$ $10^{6}$ molecules $\mathrm{cm}^{-3}$ in excited states $\left(v^{\prime \prime}=1-7\right)$ and $\sim 2.1 \times$ $10^{7}$ molecules $\mathrm{cm}^{-3}$ formed by whatever mechanism in $v^{\prime \prime}=0$ of the ground state. Some of them might be released immediately in the gas phase and some stick to the surface. The net result is that $\sim 30 \%$ of the $\mathrm{D}_{2}$ molecules are formed in excited states, $13.5 \%$ in the $v^{\prime \prime}=4$, all $J^{\prime \prime}$ state and $3.5 \%$ in the $v^{\prime \prime}=4$, $J^{\prime \prime}=2$ excited state immediately during deposition. Even if this is only a rough estimate, we find that more than $1 / 3$ of $D_{2}$ molecules are formed on a silicate surface at $12 \mathrm{~K}$ through the $\mathrm{H}-\mathrm{K}$ mechanism either in an excited or ground state.

Finally, we look at the mechanisms of $\mathrm{H}_{2}$ formation when the REMPI yield is substantially higher than the QMS-TPD. At steady state, the number of atoms on the surface is $d N / d t=$ $0=F-W N$, where $F$ is the flux, $W=v \times \exp \left(-E_{\mathrm{des}} / k T\right)$ is the desorption rate, $v=10^{12} \mathrm{~Hz}$ is the atom-surface fundamental vibration frequency and $E_{\mathrm{des}}$ is the desorption energy of an atom, taken to be $44 \mathrm{meV}$ from fitting rate equations to $\mathrm{H}_{2}$ formation data on amorphous silicates (Perets et al. 2007). We get $N \sim 1 \times 10^{7} \mathrm{~cm}^{-2}$. The rms distance traveled by an atom is $\left(\left\langle R^{2}\right\rangle\right)^{1 / 2}=4 D t$, where $D$ is the diffusion constant $\left(v a^{2} / 4\right.$ $\left.\exp \left(-E_{\text {diff }} / k T\right)\right), a$ is a typical atom's single jump distance, and $E_{\text {diff }}=35 \mathrm{meV}$ is the single atom diffusion energy on an amorphous silicate (Perets et al. 2007). This distance $\left(\left\langle R^{2}\right\rangle\right)^{1 / 2} \sim 20 \AA$ at $T=36 \mathrm{~K}$ is much smaller than the inter- particle distance, $\sim(N)^{-1 / 2}=3 \times 10^{4} \AA$. Therefore, the L-H mechanism can contribute only negligibly to $\mathrm{H}_{2}$ formation at this temperature, and another mechanism has to be invoked. Because of the mass mismatch between the $\mathrm{H}$ atom and the solid, the atom can travel quite a distance before it becomes equilibrated with the surface. Theoretical calculations of the distance traveled by a hydrogen atom landing on the surface of amorphous ice, arguably much rougher than the current amorphous silicate film, via the hot-atom mechanism yields $\sim 10-160 \AA$ depending on the surface temperature and kinetic energy of the particle.

\section{DISCUSSION AND CONCLUSIONS}

Although both rate equations derivations (Katz et al. 1999) based on experiments on silicates (Perets et al. 2007; Vidali et al. 2007) or theoretical work on graphite (Cuppen \& Hornekaer 2008; Gavardi et al. 2009) show that the window of high efficiency for $\mathrm{H}_{2}$ formation is extended and shifted to higher dust temperature with respect to crystalline surfaces, the upper range does not go much beyond $18 \mathrm{~K}$. However, there are regions in the ISM where temperatures can be higher, for example, in PDRs. In this work, we find that the detection of rovibrationally hot $\mathrm{D}_{2}$ formed on the surface of a silicate extends well beyond that range. While the formation of $\mathrm{H}_{2}$ at low temperature $(<30 \mathrm{~K})$ is well explained by the L-H reaction (Morisset et al. 2004, 2005), at higher temperature the residence time of $\mathrm{H}$ atoms is too short. At $36 \mathrm{~K}$, the rms diffusion path is $2 \mathrm{~nm}$, but the coverage is negligible since the residence time is $\sim 10^{-7} \mathrm{~s}$. Therefore, the formation of $\mathrm{D}_{2}$ can only occur if $\mathrm{D}$ atoms travel a significant distance to find other atoms that just landed on the surface (the use of D atoms prevents the contribution of atoms from a hypothetically hydrogenated surface). We propose that the $\mathrm{H}-\mathrm{K}$ mechanism provides an explanation for the formation of $\mathrm{D}_{2}$ at high surface temperature. From Figure 4 we clearly see that the decay rate of the yield with surface temperature is different for the QMS versus the REMPI signals. Therefore, the new and innovative picture that emerges is that there is simultaneous formation of $\mathrm{D}_{2}$ via the $\mathrm{L}-\mathrm{H}$ and the $\mathrm{H}-\mathrm{K}$ mechanisms. The former leads to formation of molecules that remain on the surface, indicating, as it has been amply observed in experiments (Roser et al. 2003; Hornekær et al. 2003; Congiu et al. 2009), that the mechanism of transfer of the energy gained in the formation of the molecule is quickly taken by the substrate. The H-K mechanism leads to the formation of rovibrationally hot $\mathrm{D}_{2}$ and the two coexist in the temperature range explored (5-70 K). In the preliminary analysis presented in the previous section, we find that more than one third of $\mathrm{D}_{2}$ molecules are formed on a silicate surface at $12 \mathrm{~K}$ through the $\mathrm{H}-\mathrm{K}$ mechanism either in an excited or ground state. However, while above $T \sim 18-28 \mathrm{~K}$ the $\mathrm{L}-\mathrm{H}$ mechanism produces few molecules because of the short residence times, rovibrational hot molecules are still detected (see Figure 4). In our interpretation, it is the H-K mechanism that provides the fast motion that allows atoms to find each other, overcoming the constraint of the short residence time. Calculations should be done (as for $\mathrm{H}$ diffusion on amorphous ice (Masuda et al. 1998; Al-Halabi \& Van Dishoeck 2007; Matar et al. 2008; Watanabe et al. 2010; Matar et al. 2010)) to check this. Given the recent discovery on how $\mathrm{H}$ atoms interact on the surface of graphite (Hornekær et al. 2006), there might be other mechanisms at play.

These results should be of major interest to modelers of the dynamics of infall of an interstellar cloud as well as to theoreticians working on models of the chemical evolution of a cloud, where the inclusion of gas-surface processes 
is recognized to be important (Herbst 2001; Röllig et al. 2007).

Finally, we comment on the non-detection of $\mathrm{H}_{2}$ formation pumping in the ISM that our results should explain. A reason for that is that so far no place has been found where $\mathrm{H}_{2}$ excitation due to formation pumping could be separated in the line of sight from others dominant processes, radiative and collisonal pumping. These processes are searched either from groundbased telescopes in the near infrared and particularly in the $K$-band (1.8-2.5 $\mu \mathrm{m}$ ) or from space in the 5-28 $\mu \mathrm{m}$ range (for transitions between rotational levels in the lower $v^{\prime \prime}=0$ ground state). According to our findings, emission lines starting from $v^{\prime}=4$ to lower $v^{\prime \prime}$ will have to be searched in the $X, J$, or $H$ (resp. $1 / 1.25 / 1.65 \mu \mathrm{m}$ ) infrared bands of atmospheric windows. In the case of diffuse or dark clouds, depending on the stage of star formation, one needs to look for places with temperature in the 5-60 K range and where silicates are still bare. Furthermore, they could be observed only in restricted and narrow regions in molecular clouds, most likely edge-on, because forbidden transitions require large column density in the line of sight. The advent of the new generation of giant telescopes (including adaptive optics) giving access to spatial resolution down to a few milliarcsecond in the spectroscopic mode will lead to the disentangling of this observational puzzle. This is not exclusive of multiwavelength studies with Herschel and ALMA for simultaneous $\mathrm{H}_{2}$ observations and dust characterization as in the case of NGC6720 (van Hoof et al. 2010).

Funding was provided by ANR (Agence Nationale de la Recherche; contract 07-BLAN-0129), Conseil Régional d'Ile de France (SESAME contract I-07-597R), by Conseil Général du Val d'Oise and through the European Community FP7-ITNMarie-Curie Programme (grant agreement no. 238258). G.V. is supported by NSF-Astronomy \& Astrophysics Division, grant no. 0908108 .

\section{REFERENCES}

Accolla, M., et al. 2010, Phys. Chem. Chem. Phys., submitted (arXiv:1011.5344) Al-Halabi, A., \& van Dishoeck, E. F. 2007, MNRAS, 382, 1648

Amiaud, L. 2006, PhD thesis, Univ. Cergy-Pontoise \& Observatoire de Paris, http://www.u-cergy.fr/LERMA-LAMAP/theses/Lionel_AMIAUD_2006. pdf

Amiaud, L., Dulieu, F., Fillion, J.-H., Momeni, A., \& Lemaire, J. L. 2007, J. Chem. Phys., 127, 709

Baouche, S. 2004, PhD thesis, Univ. Paris VI \& Observatoire de Paris, http://www.u-cergy.fr/LERMA-LAMAP/theses/Saoud_BAOUCHE_ 2004.pdf

Cazaux, S., \& Tielens, A. G. G. M 2004, ApJ, 604, 222

Congiu, E., Matar, E., Kristensen, L. E., Dulieu, F., \& Lemaire, J. L 2009, MNRAS, 397, L96

Cuppen, H., \& Hornekaer, L. 2008, J. Chem. Phys., 128, 174707
Davoisne, C., et al. 2008, A\&A, 482, 541

Djouadi, Z., D’Hendecourt, L., Leroux, H., Jones, A. P., Borg, J., Deboffle, D., \& Chauvin, N. 2005, A\&A, 440, 179

Fantz, U., \& Wunderlich, D. 2004, IAEA, Nuclear Data Section, Report No. 457, http://www-amdis.iaea.org/data/INDC-457

Gavardi, E., Cuppen, H., \& Hornekaer, L. 2009, Chem. Phys. Lett., 477, 285

Habart, E., Boulanger, F., Verstraete, L., Walmsley, C. M., \& Pineau des Forêts, G. 2004, A\&A, 414, 531

Harris, J., \& Kasemo, B. 1981, Surf. Sci. Lett., 105, 281

Herbst, H. 2001, Chem. Soc. Rev., 30, 168

Hollenbach, D., \& Salpeter, E. E. 1971, ApJ, 163, 155

Hornekær, L., Baurichter, A., Petrunin, V. V., Field, D., \& Luntz, A. C. 2003, Science, 302, 1943

Hornekær, L., et al. 2006, Phys. Rev. Lett., 96, 156104

Islam, F., Latimer, E. R., \& Price, S. D. 2007, J. Chem Phys., 127, 064701

Kammler, Th., Kolovos-Vellianitis, D., \& Kuppers, J. 2000, Surface Sci., 460, 91

Katz, N., Furman, I., Biham, O., Pirronello, V., \& Vidali, G. 1999, ApJ, 522, 305

Latimer, E. R., Islam, F., \& Price, S. D. 2008, Chem. Phys. Lett., 455, 174

Lemaire, J. L., \& Field, D. 2001, ESO Observation Program 66.C-0526(A) on Barnard 68, http://archive.eso.org/eso/eso_archive_main.html

Malmasson, D. 1994, PhD thesis, Univ. Paris XI \& Observatoire de Paris http://www.u-cergy.fr/LERMA-LAMAP/theses/Denis_MALMASSON_ 1994.pdf

Manicò, G., Ragunì, G., Pirronello, V., Roser, J. E., \& Vidali, G. 2001, ApJ, 548, L253

Masuda, K., Takahashi, J., \& Mukai, T. 1998, A\&A, 330, 773

Matar, E. 2009, PhD thesis, Univ. Cergy-Pontoise \& Observatoire de Paris (http://www.u-cergy.fr/LERMA-LAMAP/theses/Elie_MATAR_2009.pdf)

Matar, E., Bergeron, H., Dulieu, F., Chaabouni, H., Accolla, M., \& Lemaire, J. L. 2010, J. Chem Phys., 133, 104507

Matar, E., Congiu, E., Dulieu, F., Momeni, A., \& Lemaire, J. L. 2008, A\&A, 429, L17

Mennella, V. 2008, ApJ, 684, L25

Morisset, S., Aguillon, F., Sizun, M., \& Sidis, V. 2004, J. Chem. Phys., 121, 6493

Morisset, S., Aguillon, F., Sizun, M., \& Sidis, V. 2005, J. Chem. Phys., 122 4702

Perets, H. B., et al. 2007, ApJ, 661, L163

Pirronello, V., Liu, C., Shen, L., \& Vidali, G. 1997, ApJ, 475, L69

Röllig, M., et al. 2007, A\&A, 467, 187

Roser, J. E., Swords, S., Vidali, G., Manicò, G., \& Pirronello, V. 2003, ApJ, 596, L55

Schermann, C., Pichou, F., Landau, M., Cadez, I., \& Hall, R. I. 1994, J. Chem Phys., 101, 8152

Thi, W.-F., van Dishoeck, E. F., Bell, T., Viti, S., \& Black, J. 2009, MNRAS, 400,622

Tiné, S., Williams, D. A., Clary, D. C., Farebrother, A. J., Fisher, A. J., Meijer, A. J. H. M., Rawlings, J. M. C., \& Davis, C. J. 2003, Astrophys. Space Sci., 288,377

van Hoof, P. A. M., et al. 2010, A\&A, 518, L137

Vidali, G., Li, L., Roser, J. E., \& Badman, R. 2009, Adv. Space Res., 43, 1291

Vidali, G., et al. 2007, J. Phys. Chem. A, 111, 12611

Watanabe, N., Kimura, Y., Kouchi, A., Chigai, T., Hama, T., \& Pirronello, V. 2010, ApJ, 714, L233

Yabushita, A., Hama, T., Iida, D., Kawanaka, N., Kawasaki, M., Watanabe, N., Ashfold, M. N. R., \& Loock, H. P. 2008, ApJ, 682, L69

Zecho, T., Güttler, A., Sha, X., Lemoine, D., Jackson, B., \& Küppers, J. 2002, Chem. Phys. Lett., 366, 188 